\title{
Chondrogenic differentiation of stem cells in human umbilical cord stroma with PGA and PLLA scaffolds
}

\author{
Liang Zhao ${ }^{1,2}$, Michael S. Detamore ${ }^{1,3}$ \\ ${ }^{1}$ Department of Spinal \& Orthopaedic Surgery, Southern Medical University, Nanfang Hospital, Guangzhou, China; \\ ${ }^{2}$ Department of Chemical \& Petroleum Engineering, University of Kansas, Lawrence, KS, USA; \\ ${ }^{3}$ Department of Mechanical Engineering, University of Kansas, Lawrence, KS, USA. \\ Email: detamore@ku.edu
}

Received 9 September 2010; revised 25 September 2010; accepted 30 September 2010.

\begin{abstract}
The stem cells in the umbilical cord stroma, or Wharton's jelly, are referred to as human umbilical cord mesenchymal stromal cells (hUCMSCs) and have been shown to differentiate along a chondrogenic lineage. The aim of this study was to evaluate the chondrogenic differentiation of hUCMSCs in either polyglycolic acid (PGA) or poly-L-lactic acid (PLLA) non-woven mesh scaffolds for cartilage tissue engineering. PGA is widely known to degrade faster than PLLA, and over longer time scales, and differences may be expected to emerge after extended culture periods. Therefore, the focus of this study was to evaluate differences over a shorter duration. After 21 days of culture in PLLA or PGA scaffolds, hUCMSC constructs were analyzed for biochemical content, histology, and gene expression. Overall, there were only minute differences between the two scaffold groups, with similar gene expression and biosynthesis. The most notable difference was a change in shape from cylindrical to spherical by the PGA, but not PLLA, scaffold group. The overall similar behavior of the groups may suggest that in vivo application of hUCMSC-seeded PLLA or PGA scaffolds, following a 21-day pre-culture period, may yield similar constructs at the time of implantation. However, differences may begin to become more apparent with in vivo performance following implantation, or with in vitro performance over longer time periods.
\end{abstract}

Keywords: Umbilical Cord; Stem Cells; Cartilage Tissue Engineering; Scaffold

\section{INTRODUCTION}

The treatment of articular cartilage injuries is a challenge due to the very limited capacity for cartilage self-repair and the limited surgical techniques that successfully treat the damaged cartilage [1-3]. Stem cell-based tissue engineering techniques have the potential to revolutionize the ability to regenerate damaged cartilage [4-7].

Recently, a promising stem cell source residing in the Wharton's jelly of the umbilical cord, referred to as human umbilical cord mesenchymal stromal cells (hUCMSCs), appears to bear multi-potential mesenchymal stem cell characteristics and can differentiate into cells resembling adipocytes, osteoblasts, chondrocytes, neurons, and endothelial cells [8-15]. Recently, our laboratory has successfully induced hUCMSCs into osteogenic and chondrogenic lineages [14-16]. For engineering articular cartilage implants, a crucial consideration is the scaffolding biomaterial. These biomaterials have included a variety of natural gels and hydrogels based on collagen, glycosaminoglycans, hyaluronic acid, agarose, alginate and gelatin [17-22], as well as a number of synthetic materials used as scaffolds for chondrogenic differentiation of stem cells, such as polyglycolic acid (PGA), among the most common materials for cartilage tissue engineering [23-25]. However, previous studies showed that PGA scaffolds are limited by rapid degradation in vitro [2630].

Therefore, the aim of this study was to investigate the incorporation of hUCMSCs with either PGA or PLLA scaffolds under chondrogenic differentiation, and to compare the relative performance of the two biomaterials under these conditions.

\section{MATERIALS AND METHODS}

\subsection{Isolation and Culture of hUCMSCs}

The hUCMSCs were harvested following our previous method, with IRB approval (KU-Lawrence no.15402, KU Medical Center no.10951) and informed consent [14]. Four cords ( 2 males and 2 females, length: $20 \pm 3$ $\mathrm{cm}$ ) were obtained from the University of Kansas Medi- 
cal Center (KUMC) and were processed within 24 hours. In brief, isolated hUCMSCs were cultured in a complete culture medium consisting of low-glucose Dulbecco's modified Eagle's medium (DMEM-LG), 10\% MSCqualified fetal bovine serum (FBS), and $1 \%$ penicillin/streptomycin (PS) (Invitrogen, Carlsbad, CA). Cells were plated in cell culture flasks at 3000 cells $/ \mathrm{cm}^{2}$ (P0 cells). Non-adherent cells were rinsed off 3 days after plating. When attached hUCMSCs reached $80 \%$ confluence, the cells were detached using $0.05 \%$ trypsin-EDTA (Invitrogen) and expanded (P1). Culture medium was changed every 2 days, and the cells were split approximately $1: 4$ at each passage thereafter. Passage 4 (P4) hUCMSCs were used for all experiments.

\subsection{Identification of hUCMSCs}

The P4 hUCMSCs were characterized by flow cytometry to analyze the specific surface antigens of cells including CD13, CD29, CD34, CD45, CD49e, CD73, $\mathrm{CD} 90$ and CD105. All supplies were purchased from BD Biosciences (San Jose, CA), except CD73 and CD105 from eBioscience (San Diego, CA). In brief, approximately $0.5 \times 10^{6}$ cells per vial were used to stain. The nonspecific binding was blocked with a staining buffer including PBS and 2\% FBS for 15 minutes on ice, while the cells were incubated with single label antigen for 20 minutes on ice. Mouse isotype antigens served as the control. The analysis was measured using a FACscan (Becton Dickinson, San Jose, CA).

\subsection{Seeding hUCMSCs Into PGA and PLLA Scaffolds}

Non-woven PGA and non-woven PLLA meshes (50 $\mathrm{mg} / \mathrm{cc} ;>95 \%$ porosity; Biomedical Structures LLC, RI) were punched to create cylindrical scaffolds $(5 \mathrm{~mm}$ diameter, $2 \mathrm{~mm}$ thick). Scaffolds were sterilized following our previous methods with ethylene oxide [14]. The P4 hUCMSCs were seeded at a cell density of $25 \times 10^{6}$ cells per ml, $0.98 \times 10^{6}$ of hUCMSCs in $35 \mu \mathrm{l}$ of the complete medium were seeded in a dropwise manner into each scaffold. Cell-seeded scaffolds were incubated in a cell culture incubator for 3 hours to allow for cell attachment. To keep constructs hydrated, $10 \mu \mathrm{l}$ of complete medium was added every 1 hour. After the 3-hour period, $2 \mathrm{ml}$ of either chondrogenic medium was added into each culture well. Chondrogenic medium consisted of high-glucose DMEM (DMEM-HG; Invitrogen), $1 \%$ non-essential amino acids (NEAA; Invitrogen), $1 \mathrm{X}$ sodium pyruvate (Invitrogen), $1 \mathrm{X}$ insulin transferring selenium premix (ITS premix; BD Biosciences), $50 \mu \mathrm{g} / \mathrm{ml}$ ascorbic acid 2-phosphate (AA2P; Sigma, St Louis, MO), $40 \mu \mathrm{g} / \mathrm{ml}$ L-proline (Sigma), $100 \mathrm{nM}$ dexamethasone (Sigma) and $10 \mathrm{ng} / \mathrm{ml}$ transforming growth factor beta-1 (TGF- $\beta 1$;
R\&D system, Minneapolis, MN). For the control, a group of cell-seeded scaffolds were cultured in the complete culture medium. All of the medium was changed every two days for 21 days [15].

\subsection{Adhesion Assay}

Approximately 200,000 P4 hUCMSCs were suspended in $30 \mu \mathrm{l}$ of the complete culture medium and then seeded in the PGA and PLLA scaffolds as previously described $[15,31]$. Seeded scaffolds were incubated in a humidified atmosphere containing $5 \% \mathrm{CO}_{2}$ at $37^{\circ} \mathrm{C}$ for 6 hours to allow for cell attachment. At three and six hours, four scaffolds were rinsed with $2 \mathrm{ml}$ phosphate-buffered saline (PBS) and the cells in PBS were counted. By subtracting the number of washed out hUCMSCs from 200,000 cells per scaffold, the number of cells adhering to the scaffold was calculated [27].

\subsection{Biochemical Assays}

At 0,14 and 21 days, PGA and PLLA scaffolds were processed for biochemical assays including DNA, glycosaminoglycan (GAG) and hydroxyproline (HYP) content as in our previous work [15]. To measure DNA, GAG, and HYP contents, four scaffolds per group and time point were digested in $1.5 \mathrm{ml}$ papain solution (120 $\mu \mathrm{g} / \mathrm{ml})$ at $60^{\circ} \mathrm{C}$ overnight. DNA contents were fluorometrically determined using a PicoGreen kit according to the manufacturer's instruction (Invitrogen). GAG contents were measured using a dimethylmethylene blue (DMMB) dye binding assay kit according to the manufacturer's instructions (Biocolor, Belfast, UK). In brief, $100 \mu$ solution of each sample was measured after binding with $1 \mathrm{ml}$ of DMMB by using chondroitin sulfate as the standard. Solutions were analyzed at $656 \mathrm{~nm}$ using a Fluoroskan Ascent plate reader (Thermo Electron Corporation, Waltham, MA). HYP content was determined using a modified HYP assay protocol [15]. In brief, $400 \mu \mathrm{l}$ of specimen solution was hydrolyzed and neutralized, then analyzed at $550 \mathrm{~nm}$ using a Fluoroskan Ascent plate reader (Thermo Electron Corporation).

\subsection{Histology and Immunohistochemistry}

At 14 and 21 days, PGA and PLLA scaffolds were frozen and sectioned at a thickness of $10 \mu \mathrm{m}$ using a cryostat (Microm, Thermo Scientific, Waltham, MA). For immunohistochemical analysis, two scaffolds were used to detect types I and II collagen and aggrecan using a BioGenex 16000 autostainer (BioGenex, San Ramon, CA) as in our previous study [15]. Primary antibodies included the mouse monoclonal IgG anti-collagen type I (1:1500 dilution; Accurate Chemical and Scientific, Westbury, NY), mouse monoclonal IgG anti-collagen 
type II (1:1000 dilution; Chondrex, Redmond, WA), and mouse monoclonal IgG anti-aggrecan (1:50 dilution; Abcam, Cambridge, MA). In brief, endogenous peroxidase activity was inhibited using $1 \%$ hydrogen peroxide, blocked in horse serum and incubated with a primary antibody. Then the sections were incubated with a streptavidin-linked horse anti-mouse $\operatorname{IgG}$ secondary antibody (Vector Laboratories, Burlingame, CA). After secondary antibody incubation, the sections were incubated with an avidin-biotinylated enzyme complex (ABC complex; Vector Laboratories) and VIP substrate (Vector Laboratories). Histological analyses were performed using Safranin $\mathrm{O} /$ fast green staining with Harris hematoxylin (Sigma) to visualize GAG distribution [32].

\subsection{Quantitative Real-Time Reverse Transcription Polymerase Chain Reaction}

Total RNA of PGA and PLLA scaffolds were extracted with TRIzol reagent according to the manufacturer's protocol (Invitrogen) and reverse-transcribed into cDNA using a high-capacity cDNA Archive kit (Applied Biosystems, Foster city, CA). Real-time RT-PCR reactions were performed using an Applied Biosystems 7500 Fast System. TaqMan gene expression assay kits (Applied Biosystems), including two pre-designed specific primers and probes, were used to measure the transcript levels of the proposed genes including human type I collagen (Hs00164004), type II collagen (Hs00156568), aggrecan (Hs00153936) and glyceraldehyde 3-phosphate dehydrogenase (GAPDH; Hs99999905). Relative expression level for each target gene was evaluated using the $2^{-\Delta \Delta \mathrm{Ct}}$ method [33]. The control samples at the first day served as the calibrator. The fold of change was obtained with $\mathrm{n}=4$.

\section{RESULTS}

\subsection{Phenotype of hUCMSCs}

The P4 hUCMSCs were characterized with respect to the expression of surface antigens by flow cytometry. The hUCMSCs that were characterized with respect to the expressions of CD34 and CD45 were less than $1 \%$, whereas hUCMSCs had positive expressions for CD73 (96 $\pm 2 \%)$, CD90 $(98 \pm 4 \%)$ and CD105 (99 $\pm 3 \%)$. The hUCMSCs also expressed high levels of CD13 (96 \pm $4 \%), \mathrm{CD} 29$ (95 $\pm 4 \%$ ) and CD49e (98 4\%) (Figure 1).

\subsection{Morphology and Adhesion Assay of PGA and PLLA Scaffolds}

After 21 days of chondrogenic differentiation culture, the shapes of PGA and PLLA scaffolds were observed to be different. The shape of PGA scaffolds significantly changed from cylindrical to spherical over the 21 day period (Figures 2(a), (c)). The contraction of PLLA scaffolds was relatively negligible during the same process and no significant change of the PLLA scaffolds' morphology was observed (Figures 2(b), (d)).

For the adhesion assay, three hours after seeding hUCMSCs, the adherent cell percentage was $60 \pm 9 \%$ in PGA scaffolds and was $58 \pm 6 \%$ in PLLA scaffolds. After 6 hours, the adherent cell percentage increased to 73 $\pm 8 \%$ in PGA scaffolds and to $75 \pm 4 \%$ in PLLA scaffolds. The results from the adhesion assay showed no significant difference between PGA and PLLA scaffolds $(\mathrm{p}>0.1)$ (Figure 2(e)).

\subsection{Biochemical Assays}

Throughout the entire period of the chondrogenic differentiation culture, the DNA content decreased in both scaffolds as shown in Figure 3(a). The DNA content decreased approximately $30 \%$ in the PGA scaffolds and $21 \%$ in the PLLA scaffolds over the 21 day period. At 21 days, the DNA content in PLLA scaffolds was $18.8 \%$ higher than that of PGA scaffolds $(\mathrm{p}<0.05)$.

The GAG content in both scaffolds decreased with culture time (Figure 3(b)). In the PGA scaffolds, the highest GAG content of $11.1 \pm 2.4 \mu \mathrm{g} / \mathrm{scaffold}$ was measured at 0 days. The GAG content in the PGA scaffolds decreased by $51 \%$ at 14 days and decreased by $80 \%$ at 21 days when compared to the 0 day values. In the PLLA scaffolds, the highest GAG content was $10.9 \pm$ $2.3 \mu \mathrm{g} / \mathrm{scaffold}$ at 0 days. The GAG content in PLLA scaffolds decreased by $43 \%$ at 14 days and decreased by $70 \%$ at 21 days when compared to the 0 day values. At 21 days, the GAG content in PLLA scaffolds was higher than that in PGA scaffolds $(p<0.05)$.

Cumulative HYP production in the PGA and PLLA scaffolds was measured at 0,14 , and 21 days. The higher HYP content was observed at 14 days in both scaffolds. In the PGA scaffold group, the HYP content was $1.5 \pm$ $0.1 \mu \mathrm{g} / \mathrm{scaffold}$ at 14 days and $1.2 \pm 0.1 \mu \mathrm{g} / \mathrm{scaffold}$ at 21 days. In the PLLA scaffold group, the HYP content was $1.4 \pm 0.2 \mu \mathrm{g} / \mathrm{scaffold}$ at 14 days and $1.1 \pm 0.1 \mu \mathrm{g} / \mathrm{scaffold}$ at 21 days. The HYP content measurements showed no significant difference between the HYP contents of the PGA and PLLA scaffolds $(\mathrm{p}>0.1)$ (Figure 3(c)).

\subsection{Histology and Immunohistochemistry}

At 14 days, both PGA and PLLA sections showed a moderate amount of collagen type I staining with a trace amount of collagen type II and aggrecan staining. There was no significant difference in staining intensity present between the PGA and PLLA groups (Figure 4). Differences in immunostaining were generally minimal between 14 days and 21 days. Only Saf-O staining appeared 

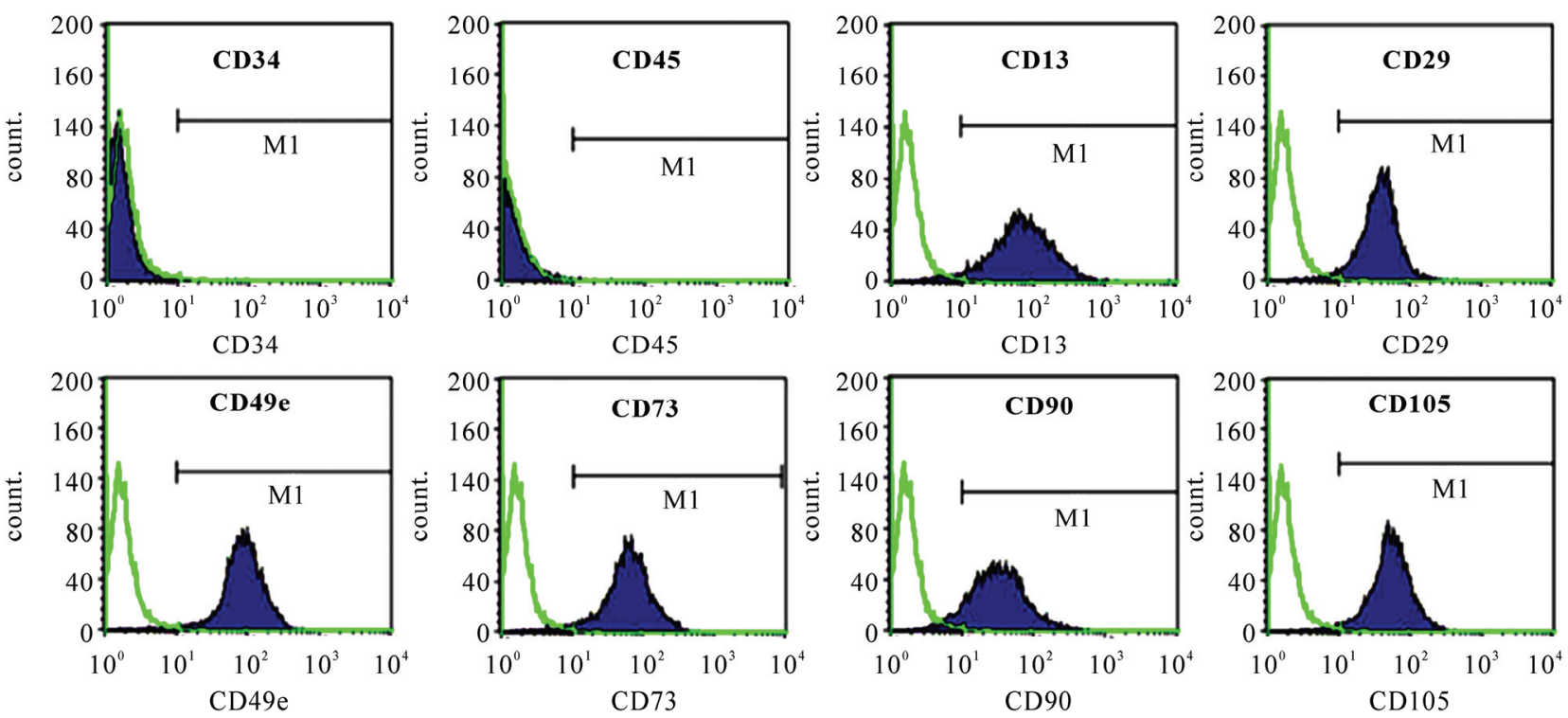

(a)

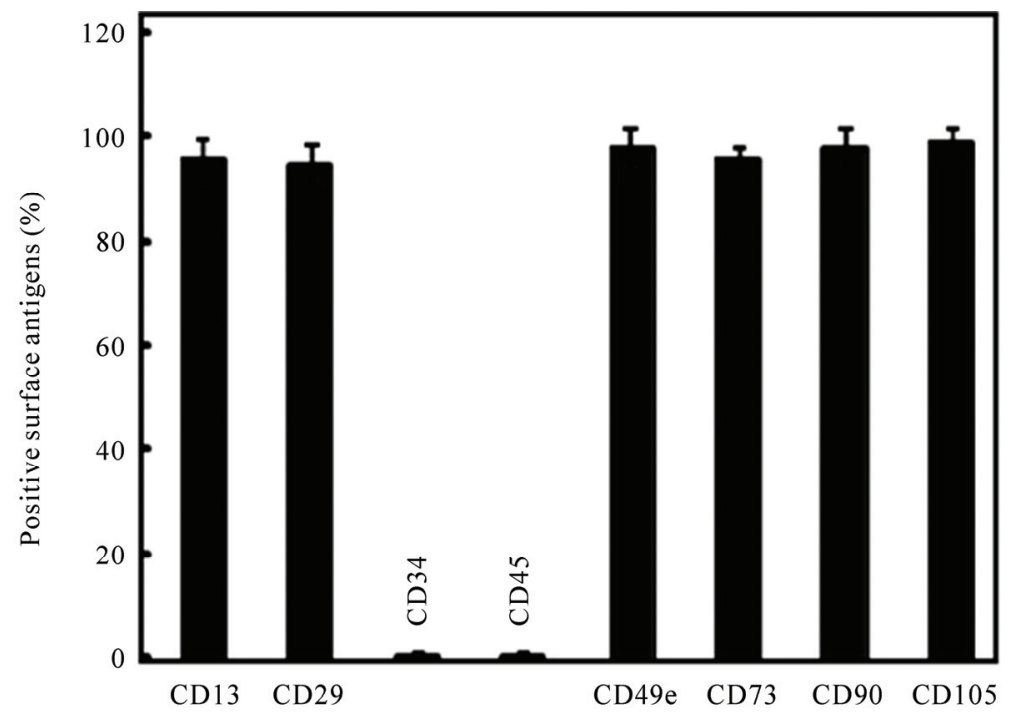

(b)

Figure 1. Flow cytometric analysis of surface-marker expression of P4 hUCMSCs. (a) Surface phenotypic characterization of hUCMSCs from a representative sample. The hUCMSCs were positive for CD13, CD29, CD49e, CD73, CD90 and CD105, but negative for CD34 and CD45; (b) The histogram plot of expression of cell surface markers (mean \pm standard deviation; $n=4$ ).

to increase in intensity from 14 days to 21 days (Figure 4).

\subsection{Gene Expression}

As shown in Figure 5, at 14 days and 21 days, the cells in both PGA and PLLA scaffolds were observed to significantly activate expression of genes encoding for collagen type I, collagen type II and aggrecan $(p<0.05)$. In PGA scaffolds, collagen I showed a 3-fold increase at 14 days and a 15 -fold increase at 21 days when compared to
0 day $(\mathrm{p}<0.05)$. The collagen II expression in PGA scaffolds increased 8-fold at 14 days and 9-fold at 21 days when compared to 0 days $(\mathrm{p}<0.05)$. Aggrecan in the PGA scaffolds increased 3-fold at 14 days and 6-fold at 21 days when compared to 0 day $(\mathrm{p}<0.05)$. In PLLA scaffolds, collagen I showed a 4-fold increase at 14 days and a 16-fold increase at 21 days when compared to 0 day $(p<0.05)$. The collagen II in PLLA scaffolds increased 7 -fold at 14 days and 10-fold at 21 days when compared to day 0 , while aggrecan expression increased 


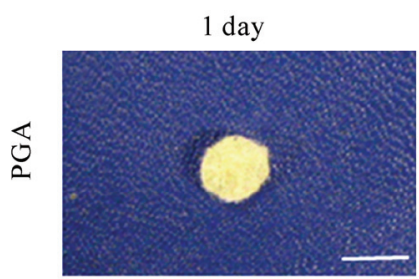

(a)

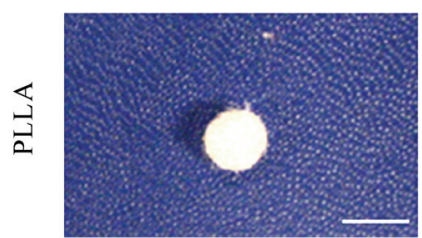

(c)

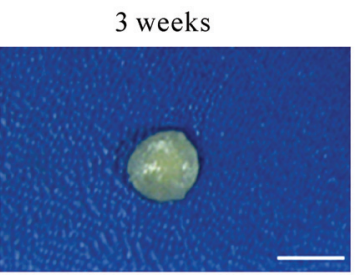

(b)

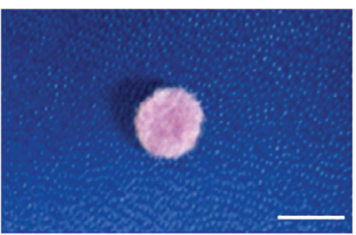

(d)

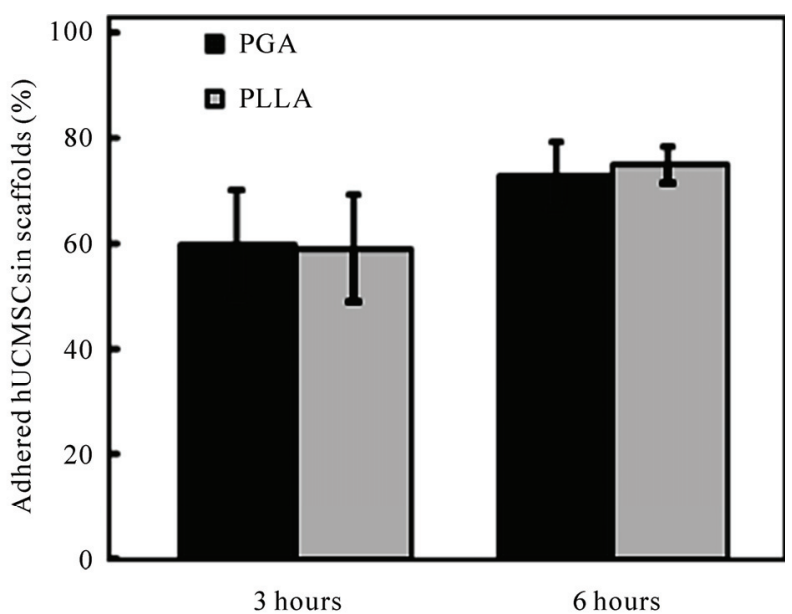

Figure 2. Morphology and adhesion assay of PGA and PLLA scaffolds. (a) The morphology of a representative PGA scaffold at 1 day after seeding; (b) The morphology of a representative PLLA scaffold at 1 day when seeded with hUCMSCs; (c) The morphology of a representative PGA scaffold after 21 days of chondrogenic differentiation culture; (d) The morphology of a representative PLLA scaffold at 21 days. The scale bars are 5 mm; (e) Adhesion assay of PGA and PLLA scaffolds (mean \pm standard deviation; $\mathrm{n}=4$ ).

3 -fold at 14 days and 5-fold at 21 days when compared to day $0(\mathrm{p}<0.05)$. However, there was no significant difference in gene expression between the PGA and PLLA scaffold groups $(\mathrm{p}>0.1)$ (Figure 5).

\section{DISCUSSION}

hUCMSCs may be considered to be a promising, inexhaustible and low-cost source of mesenchymal stem cells. In previous studies, hUCMSCs were successfully induced for chondrogenic differentiation $[15,16,30]$. The present study investigated the influence of seeding hUCMSCs into PGA and PLLA scaffolds on the potential ability of chondrogenesis in vitro. The results showed

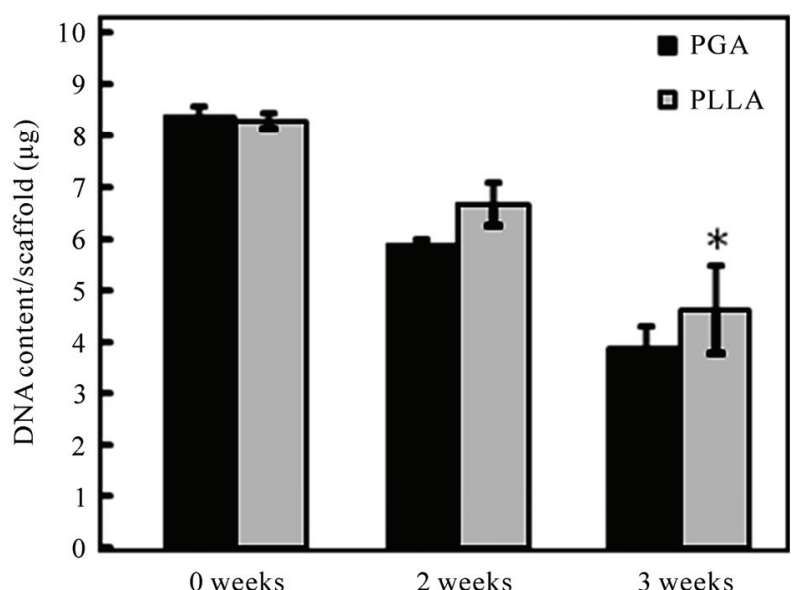

(a)

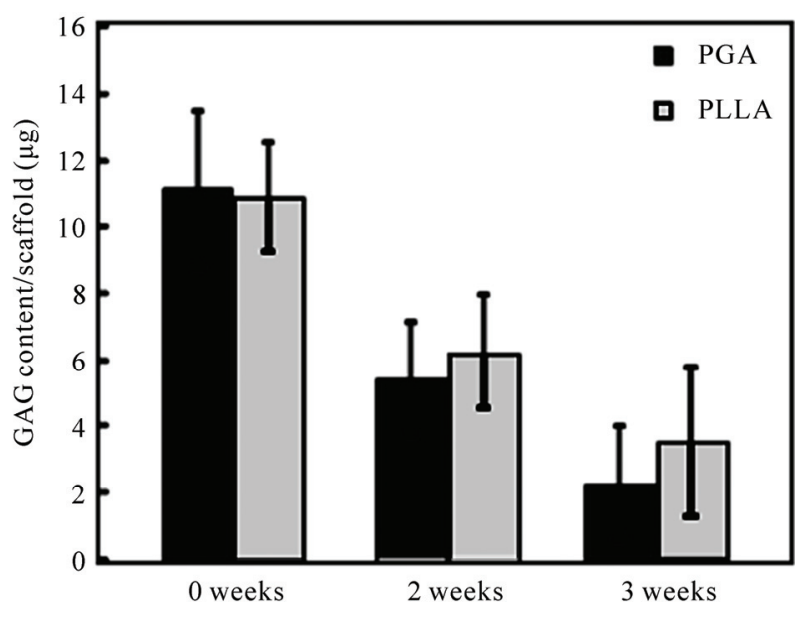

(b)

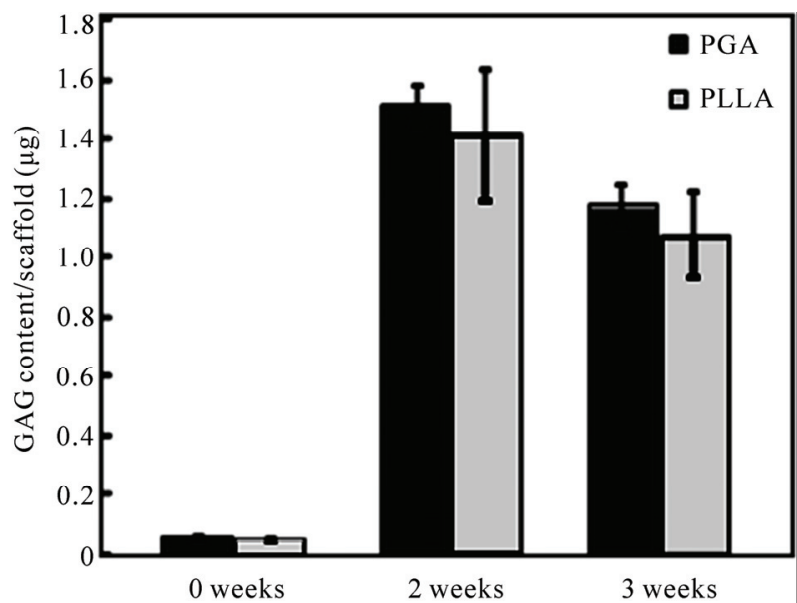

(c)

Figure 3. Biochemical analyses are shown for the chondrogenic differentiation of hUCMSCs in PGA and PLLA scaffolds (mean \pm standard deviation; $n=4$ ). (a) DNA content; (b) Glycosaminoglycan (GAG) content; (c) Hydroxyproline (HYP) content. $*=$ statistically significant difference between the PGA and the PLLA scaffolds $(\mathrm{p}<0.05)$. 

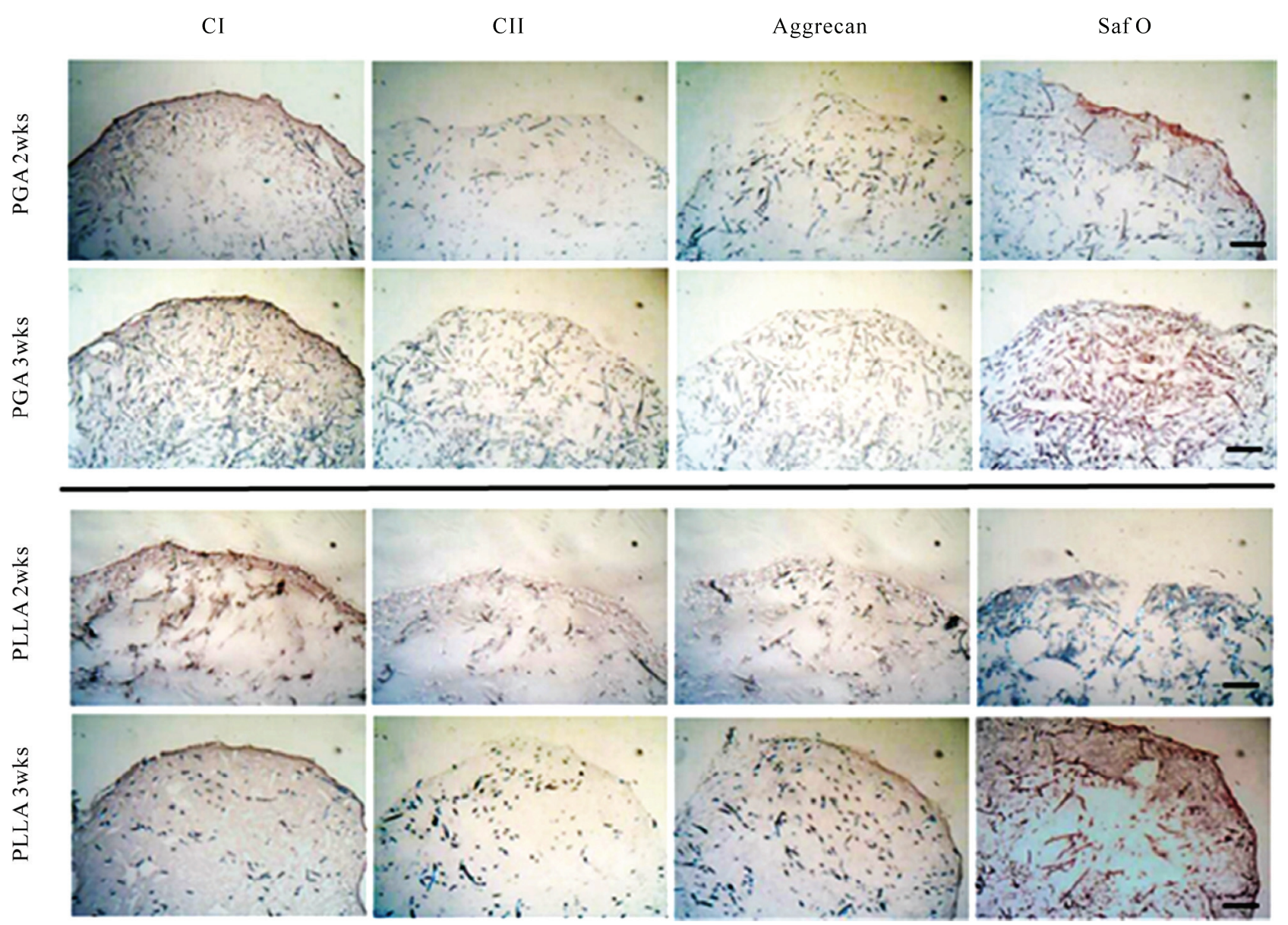

Figure 4. Immunohistochemical staining for type I and II collagen and aggrecan, and Safranin-O staining $(\mathrm{n}=2)$ at 14 days and 21 days. With chondrogenic differentiation, both the PGA and PLLA scaffolds stained faintly for cartilage-specific proteins collagen II and aggrecan. Safranin-O staining was observed in both PGA and PLLA scaffolds. The scale bar is $250 \mu \mathrm{m}$. CI = collagen type I, CII = collagen type II, Saf O = Safranin-O.

some degree of chondrogenic differentiation of hUCMSCs, as evidenced by the expression of collagen type II and aggrecan genes.

The present study showed that hUCMSCs could differentiate along a chondrogenic lineage in both PGA and PLLA scaffolds. The similar chondrogenic differentiation patterns were quantitatively characterized by the extracellular matrix, including GAG production (Figure 3(b)) and collagen production (Figure 3(c)). Histology showed staining for GAGs (Figure 4), although these GAGs did not aggregate to form a large amount of aggrecan as indicated by only a trace aggrecan staining. Quantitative RT-PCR showed the up-regulation of cartilage marker gene expressions, specifically collagen type II and aggrecan (Figure 5(b), (c)). This upregulation of aggrecan and collagen II gene expression may show potential in longer-term studies for greater cumulative levels of aggrecan and collagen II production.

The physicochemical characteristics and mechanical performances of PGA are well established in clinical practice. However, previous studies showed that PGA fibers degraded relatively quickly, losing their integrity and becoming fiber fragments in the cell culture medium as quickly as within $2-4$ weeks $[14,34-36]$. PLLA provides more space for cellular, biochemical, and even biomechanical development [37,38]. In the present study, during the 21 days chondrogenic differentiation culture, PGA scaffolds were observed to morphologically change in shape more than the PLLA scaffolds (Figure 2(c), (d)). Moreover, the DNA of the PLLA group was relatively higher than that of the PGA group after 14 days of culture (Figure 3(a)). The reason may be that the PGA scaffolds, which are expected to degrade faster, led to a greater structural shift. Our results showed that the chondrogenic potential of hUCMSCs in PLLA scaffolds was similar to that in PGA scaffolds. PLLA scaffolds showed a relatively stable morphology during the same chondrogenic differentiation conditions. 


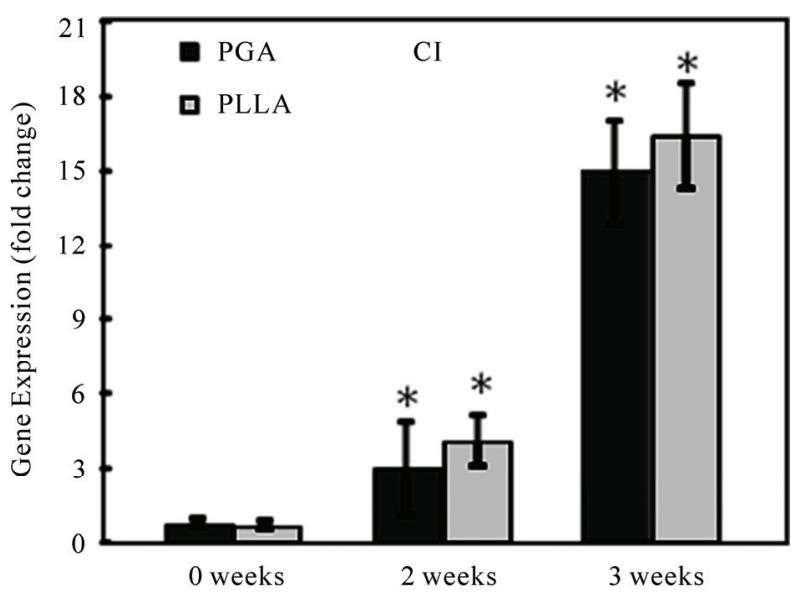

(a)

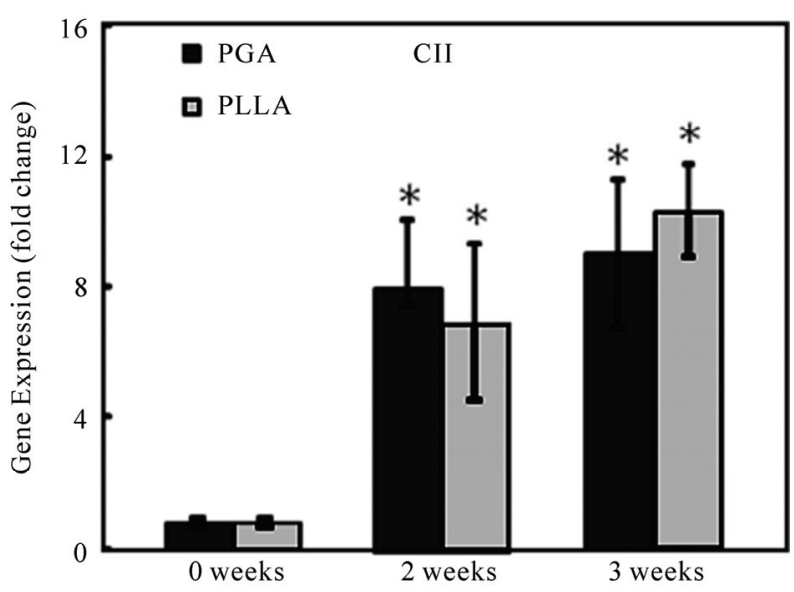

(b)

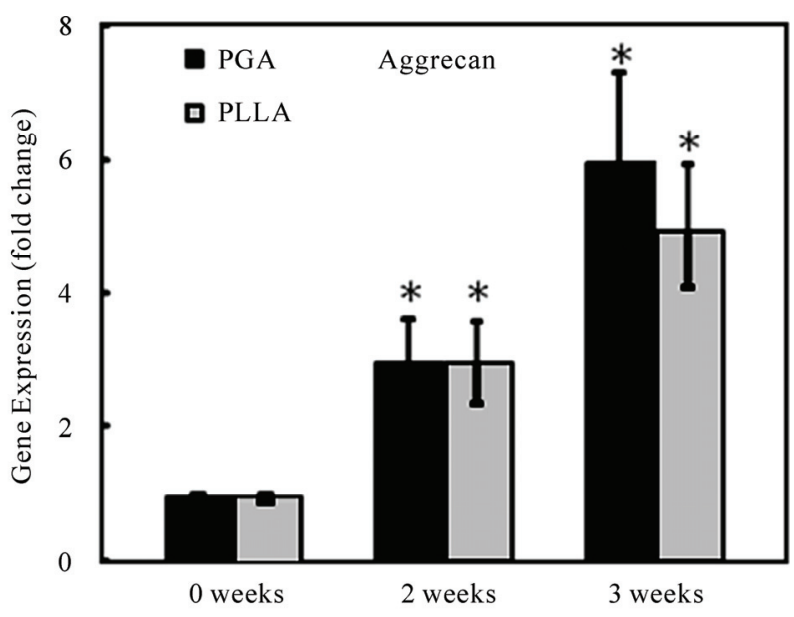

(c)

Figure 5. Quantitative relative gene expression profile of hUCMSCs in both PGA and PLLA scaffolds at 0,14 and 21 days (mean \pm standard deviation; $n=4$ ). (a) Collagen type I gene expression. (b) Collagen type II gene expression. (c) Aggrecan gene expression. $\mathrm{CI}=$ collagen type $\mathrm{I}, \mathrm{CII}=$ collagen type II. *= statistically significant difference among the PGA and the PLLA groups compared to day $0(\mathrm{p}<0.05)$.

\section{CONCLUSIONS}

In the present study, the chondrogenic differentiation of hUCMSCs in both PGA and PLLA scaffolds has been evaluated. Compared with the PGA scaffolds, PLLA scaffolds showed a relatively stable morphology during the same period ( $3 \mathrm{wks})$ and conditions. The results indicated that hUCMSCs had some degree of chondrogenic potential in both PGA and PLLA scaffolds. Differences between the PLLA and PGA scaffold groups were minimal over a 21-day period, indicating that an in vivo application requiring an in vitro pre-culture time on the order of 21 days would find these two cell-seeded biomaterials in a similar state. However, differences may begin to become more apparent in the longer term (e.g., 6 weeks).

\section{ACKNOWLEDGEMENTS}

We gratefully acknowledge Dr. Xinkun Wang for his guidance in performing the RT-PCR. We also thank Dr. Limin Wang for his RT-PCR assistance, and Lauren Byers for her assistance in proofreading the manuscript. This study was supported by NIH R21 grant DE017673-01, Arthritis Foundation (National and Kansas Chapters), and the State of Kansas.

\section{REFERENCES}

[1] Simon, T.M. and Jackson, D.W. (2006) Articular cartilage: Injury pathways and treatment options. Sports Medicine and Arthroscopy Review, 14, 146-154.

[2] Verbruggen, G., Wittoek, R., Groeneboer, S., Cruyssen, B.V., Goemaere, S. and Elewaut, D. (2007). Osteochondral repair in synovial joints. Current Opinion in Rheumatology, 19, 265-271.

[3] Hunziker, E.B. (1999) Articular cartilage repair: Are the intrinsic biological constraints undermining this process insuperable? Osteoarthritis and Cartilage/OARS, Osteoarthritis Research Society, 7, 15-28.

[4] Shay, J.W. and Wright, W.E. (2000) The use of telomerized cells for tissue engineering. Nature Biotechnology, 18, 22-23.

[5] Liechty, K.W., MacKenzie, T.C., Shaaban, A.F., Radu, A. Moseley, A.M., Deans, R., Marshak, D.R. and Flake, A.W. (2000) Human mesenchymal stem cells engraft and demonstrate site-specific differentiation after in utero transplantation in sheep. Nature Medicine, 6, 1282-1286.

[6] Yamashita, J., Itoh, H., Hirashima, M., Ogawa, M., Nishikawa, S., Yurugi, T., Naito, M., Nakao, K. and Nishikawa, S. (2000) Flk1-positive cells derived from embryonic stem cells serve as vascular progenitors. Nature, $\mathbf{4 0 8 ,}$ 92-96.

[7] Service, R.F. (2000) Tissue engineers build new bone. Science, 289, 1498-1500.

[8] Wang, H.S., Hung, S.C., Peng, S.T., Huang, C.C., Wei, H.M., Guo, Y.J., Fu, Y.S., Lai, M.C. and Chen, C.C. (2004) Mesenchymal stem cells in the Wharton's jelly of the human umbilical cord. Stem Cells, 22, 1330-1337.

[9] Weiss, M.L., Medicetty, S., Bledsoe, A.R., Rachakatla, 
R.S., Choi, M., Merchav, S., Luo, Y., Rao, M.S., Velagaleti, G. and Troyer, D. (2006) Human umbilical cord matrix stem cells: Preliminary characterization and effect of transplantation in a rodent model of Parkinson's disease. Stem Cells, 24, 781-792.

[10] Can, A. and Karahuseyinoglu, S. (2007) Concise review, human umbilical cord stroma with regard to the source of fetus-derived stem cells. Stem Cells, 25, 2886-2895.

[11] Friedman, R., Betancur, M., Boissel, L., Tuncer, H., Cetrulo, C. and Klingemann, H. (2007) Umbilical cord mesenchymal stem cells, adjuvants for human cell transplantation. Biology of Blood and Marrow Transplantation, 13, 1477-1486.

[12] Bakhshi, T, Zabriskie, R.C., Bodie, S., Kidd, S., Ramin, S., Paganessi, L.A., Gregory, S.A., Fung. H.C. and Christopherson, K.W. (2008) Mesenchymal stem cells from the Wharton's jelly of umbilical cord segments provide stromal support for the maintenance of cord blood hematopoietic stem cells during long-term ex vivo culture. Transfusion, 48, 2638-2644.

[13] Baksh, D., Yao, R. and Tuan, R.S. (2007) Comparison of proliferative and multilineage differentiation potential of human mesenchymal stem cells derived from umbilical cord and bone marrow. Stem Cells, 25, 1384-1392.

[14] Wang, L., Seshareddy K., Weiss, M.L. and Detamore, M.S. (2009) Effect of initial seeding density on human umbilical cord mesenchymal stromal cells for fibrocartilage tissue engineering. Tissue Engineering, Part A, 15, 1009-1017.

[15] Wang, L., Tran, I., Seshareddy, K., Weiss, M.L. and Detamore, M.S. (2009) A comparison of human bone marrow-derived mesenchymal stem cells and human umbilical cord-derived mesenchymal stromal cells for cartilage tissue engineering. Tissue Engineering, Part A, 15, 22592266.

[16] Wang L., Singh, M., Bonewald, L.F. and Detamore, M.S. (2009) Signalling strategies for osteogenic differentiation of human umbilical cord mesenchymal stromal cells for 3D bone tissue engineering. Journal of Tissue Engineering and Regenerative Medicine, 3, 398-404.

[17] Masuoka, K., Asazuma, T., Ishihara, M., Sato, M., Hattori, H., Ishihara, M., Yoshihara, Y., Matsui, T., Takase, B., Kikuchi, M. and Nemoto, K. (2005) Tissue engineering of articular cartilage using an allograft of cultured chondrocytes in a membrane-sealed atelocollagen honeycomb-shaped scaffold (ACHMS scaffold). Journal of Biomedical Materials Research, 75, 177-184.

[18] Chang, C.H., Kuo, T.F., Lin, C.C., Chou, C.H., Chen, K.H., Lin, F.H. and Liu, H.C. (2006) Tissue engineering-based cartilage repair with allogenous chondrocytes and gelatin-chondroitin-hyaluronan tri-copolymer scaffold, A porcine model assessed at 18, 24, and 36 weeks. Biomaterials, 27, 1876-1888.

[19] Lisignoli, G., Cristino, S., Piacentini, A., Toneguzzi, S., Grassi, F., Cavallo, C., Zini, N., Solimando, L., Mario Maraldi, N. and Facchini, A. (2005) Cellular and molecular events during chondrogenesis of human mesenchymal stromal cells grown in a three-dimensional hyaluronan based scaffold. Biomaterials, 26, 5677-5686.

[20] Mauck, R.L., Yuan, X. and Tuan, R.S. (2006) Chondrogenic differentiation and functional maturation of bovine mesenchymal stem cells in long-term agarose culture. Osteoarthritis and Cartilage/OARS, Osteoarthritis Research Society, 14, 179-189.

[21] Nettles, D.L., Elder, S.H. and Gilbert, J.A. (2002) Potential use of chitosan as a cell scaffold material for cartilage tissue engineering. Tissue Engineering, 8, 1009-1016.

[22] Steinert, A, Weber, M., Dimmler, A., Julius, C., Schutze, N., Noth, U., Cramer, H., Eulert, J., Zimmermann, U. and Hendrich, C. (2003) Chondrogenic differentiation of mesenchymal progenitor cells encapsulated in ultrahighviscosity alginate. Journal of Orthopaedic Research, 21, 1090-1097.

[23] Mahmoudifar, N. and Doran, P.M. (2005) Tissue engineering of human cartilage and osteochondral composites using recirculation bioreactors. Biomaterials, 26, 70127024.

[24] Puelacher, W.C., Mooney, D., Langer, R., Upton, J., Vacanti, J.P. and Vacanti, C.A. (1994) Design of nasoseptal cartilage replacements synthesized from biodegradable polymers and chondrocytes. Biomaterials, 15, 774-778.

[25] Ma, P.X., Schloo, B., Mooney, D. and Langer, R. (1995) Development of biomechanical properties and morphogenesis of in vitro tissue engineered cartilage. Journal of Biomedical Materials Research, 29, 1587-1595.

[26] Ishaug-Riley, S.L., Okun, L.E., Prado, G., Applegate, M.A. and Ratcliffe, A. (1999) Human articular chondrocyte adhesion and proliferation on synthetic biodegradable polymer films. Biomaterials, 20, 2245-2256.

[27] Zwingmann, J., Mehlhorn, A.T., Sudkamp, N., Stark, B., Dauner, M. and Schmal, H. (2007) Chondrogenic differentiation of human articular chondrocytes differs in biodegradable PGA/PLA scaffolds. Tissue Engineering, 13, 2335-2343.

[28] Richardson, S.M., Curran, J.M., Chen, R., VaughanThomas, A., Hunt, J.A., Freemont, A.J. and Hoyland, J.A. (2006) The differentiation of bone marrow mesenchymal stem cells into chondrocyte-like cells on poly-L-lactic acid (PLLA) scaffolds. Biomaterials, 27, 4069-4078.

[29] Stevens, M.M., Qanadilo, H.F., Langer R. and Prasad Shastri, V. (2004) A rapid-curing alginate gel system, utility in periosteum-derived cartilage tissue engineering. Biomaterials, 25, 887-894.

[30] Wang, L., Seshareddy, K., Weiss, M.L. and Detamore, M.S. (2009) Effect of initial seeding density on human umbilical cord mesenchymal stromal cells for fibrocartilage tissue engineering. Tissue Engineering, Part A, 15(5), 1009-1017.

[31] Wang, L., Dormer, N.H., Bonewald, L.F. and Detamore, M.S. (2010) Osteogenic differentiation of human umbilical cord mesenchymal stromal cells in polyglycolic acid scaffolds. Tissue Engineering, Part A, 16, 19371948.

[32] Kawanishi, M., Oura, A., Furukawa, K., Fukubayashi, T., Nakamura, K., Tateishi, T. and Ushida, T. (2007) Redifferentiation of dedifferentiated bovine articular chondrocytes enhanced by cyclic hydrostatic pressure under a gas-controlled system. Tissue Engineering, 13, 957-964.

[33] Livak, K.J. and Schmittgen, T.D. (2001) Analysis of Relative Gene Expression Data Using Real-Time Quantitative PCR and the 2- $\Delta \Delta \mathrm{CT}$ Method. Methods, 25, 402-408. 
[34] Allen, K.D. and Athanasiou, K.A. (2008) Scaffold and growth factor selection in temporomandibular joint disc engineering. Journal of Dental Research, 87, 180-185.

[35] Freed, L.E., Vunjak-Novakovic, G., Biron, R.J., Eagles, D.B., Lesnoy, D.C., Barlow, S.K. and Langer, R. (1994) Biodegradable polymer scaffolds for tissue engineering. Bio-Technology, 12, 689-693.

[36] Kang, Y., Yang, J., Khan, S., Anissian, L. and Ameer, G.A. (2006) A new biodegradable polyester elastomer for cartilage tissue engineering. Journal of Biomedical Materials Research A, 77, 331-339.
[37] Yang, F., Qu, X., Cui, W., Bei, J., Yu, F., Lu, S. and Wang, S. (2006) Manufacturing and morphology structure of polylactide-type microtubules orientation-structured scaffolds. Biomaterials, 27, 4923-4933.

[38] Rotter, N., Aigner, J., Naumann, A., Planck, H., Hammer, C., Burmester, G. and Sittinger, M. (1998) Cartilage reconstruction in head and neck surgery, comparison of resorbable polymer scaffolds for tissue engineering of human septal cartilage. Journal of Biomedical Materials Research, 42, 347-356. 